\title{
Effect of needle size on immunogenicity and reactogenicity of vaccines in infants: randomised controlled trial
}

\author{
Linda Diggle, Jonathan J Deeks, Andrew J Pollard
}

\begin{abstract}
Objectives To assess the immunogenicity of vaccines for infants and to investigate whether the incidence of reactogenicity is reduced after each immunisation dose using needles of varying lengths and gauges.

Design Randomised controlled trial.

Setting 18 general practices within two UK primary care trusts. Participants 696 healthy infants vaccinated at 2, 3, and 4 months of age, with follow-up to 5 months of age.

Interventions Combined diphtheria, tetanus, whole cell pertussis, and Haemophilus influenzae type b vaccine and a serogroup $\mathrm{C}$ meningococcal glycoconjugate vaccine administered using either a wide, long needle (23 gauge/0.6 $\mathrm{mm}$ diameter, $25 \mathrm{~mm}$ ), a narrow, short needle (25 gauge/ 0.5 $\mathrm{mm}$ diameter, $16 \mathrm{~mm}$ ), or a narrow, long needle (25 gauge, 25 $\mathrm{mm})$.
\end{abstract}

Main outcome measures Local and general reactions recorded by parents for three days after each dose; and diphtheria, tetanus, and H influenzae type b antibody concentrations and functional antibody against serogroup C Neisseria meningitidis 28-42 days after the third dose.

Results Local reactions to diphtheria, tetanus, whole cell pertussis, $H$ influenzae type b vaccinations decreased significantly with wide, long needles compared with narrow, short needles. At all three doses one less infant experienced local reactions at days 1, 2, or 3 for every six to eight vaccinated. Significantly fewer infants vaccinated with the long needle experienced severe local reactions. Non-inferiority of the immune response was shown using a wide, long needle rather than a narrow, short needle for serogroup $\mathrm{C}$ meningococcal glycoconjugate vaccine and for diphtheria but not for $H$ influenzae type b or tetanus, although no evidence was found of a decrease. Little difference was found between needles of the same length but different gauges in local reaction or immune response.

Conclusions Long $(25 \mathrm{~mm}$ ) needles for infant immunisations can significantly reduce vaccine reactogenicity at each dose while achieving comparable immunogenicity to that of short (16 mm) needles.

Trial registration Current Controlled Trials ISRCTN62032215.

\section{Introduction}

Within UK general practices infants are immunised at 2, 3, and 4 months of age. ${ }^{1}$ Although over four million injected vaccines are given to UK infants each year, ${ }^{2-5}$ evidence based guidance on needle size for intramuscular delivery is lacking. The width (gauge) of a needle is defined by hub colour. Latest Department of Health literature recommends a $25 \mathrm{~mm}$ blue hub (23 gauge) needle, ${ }^{6}$ but many practitioners prefer to use a $16 \mathrm{~mm}$ orange hub (25 gauge) needle. Some local policies stipulate use of the larger diameter 23 gauge needle, drawing on anecdotal evidence that this enables the vaccine to dissipate over a wider space, reducing risks of localised redness and swelling. ${ }^{7}$ Others advise that a $16 \mathrm{~mm}$ needle adequately reaches anterolateral thigh muscle when inserted at $90^{\circ}$ and using the intramuscular delivery technique recommended by the World Health Organization. ${ }^{8} \mathrm{WHO}$, however, recommends a $25 \mathrm{~mm}$ needle for infant immunisations. ${ }^{9}$ Uncertainty has arisen because of insufficient data to define best practice.

We have shown that wider, longer needles significantly reduce the incidence of local reaction at 4 months of age. ${ }^{10}$ This study did not, however, determine whether reduced reactogenicity was related to a difference in gauge or length and whether the same effects would be seen at earlier immunisations, nor whether needle size affected vaccine immunogenicity. Benefits gained from a reduction in local reactogenicity would have to be balanced against any compromise of immunogenicity.

We carried out a randomised controlled trial to compare three needles of varying diameter and length (23 gauge, $25 \mathrm{~mm}$; 25 gauge, $16 \mathrm{~mm}$; and 25 gauge, $25 \mathrm{~mm}$ ). We also assessed immunogenicity as well as local and general reaction rates after immunisation with combined diphtheria, pertussis, tetanus, and Haemophilus influenzae type b vaccine and a serogroup C meningococcal glycoconjugate vaccine in infants at 2, 3, and 4 months of age. The trial was designed to test whether immunogenicity using a wide, long needle is equivalent or superior to a narrow, short needle (a non-inferiority hypothesis). If the previously observed reduction in adverse events with the wider, longer needle was repeated, while achieving comparable immunogenicity, this would be sufficient to recommend best practice.

\section{Methods}

Eighteen of 35 general practices approached in the Vale of Aylesbury primary care trust, Buckinghamshire and North East Oxfordshire primary care trust agreed to recruit. Parents of infants due to receive their first immunisation between April 2002 and March 2004 were sent a letter inviting their children to participate. We excluded infants of less than 37 weeks' gestation, those of birth weight less than $2500 \mathrm{~g}$, and those undergoing treatment or with conditions that could bias the evaluation of an

Local reactions according to needle size are on bmj.com 
immune response. The first vaccination was at age 8-11 weeks, with subsequent vaccinations at intervals of 4-6 weeks.

\section{Study procedures}

Infants were allocated to a needle group according to a computer generated randomisation scheme generated by the study statistician, stratified by general practice with random variable block sizes of three, six, and nine. Allocations were concealed in sequentially numbered opaque sealed envelopes. When written parental consent had been obtained, the study nurse allocated the next sequential participant number and opened the appropriate envelope to determine to which needle size the infant had been randomised: wide, long (23 gauge, $25 \mathrm{~mm}$ ), narrow, short (25 gauge, $16 \mathrm{~mm}$ ), or narrow, long (25 gauge, $25 \mathrm{~mm}$ ) needle. Infants received all immunisations through the allocated needle size.

A combined diphtheria, pertussis, tetanus, and Haemophilus influenzae type b vaccine (ACT-Hib DTP; Pasteur-Mèrieux-MSD, Berkshire) was administered into the right anterolateral thigh, with a meningococcal $\mathrm{C}$ vaccine given concomitantly into the left thigh: 72\% of infants received Meningitec (Wyeth Vaccines, Berkshire) at the first and second doses and $76 \%$ at the third dose, and the remainder received Menjugate (Chiron Vaccines, Liverpool).

We used the standard intramuscular injection technique as advocated for infants by WHO: the vaccine was injected into the anterolateral thigh, with the skin stretched flat between the thumb and forefinger and the needle inserted at $90^{\circ}$ and pushed down into the muscle. ${ }^{9}$ Parents were not informed of the allocated needle size. As needle gauge can be identified by hub colour, parents were not informed of the significance of these differences. Parents known to be health professionals were asked not to view the injection procedure.

Study nurses instructed parents on how to complete the diary on reactions after each immunisation; parental rating of local and systemic symptoms has been validated. ${ }^{11}$ Parents measured the diameter of any redness, hardness, or swelling using a ruler printed in the diary. Infants' reactions to movement of the thigh or touch of the injection site were graded on a scale of none, mild, moderate, or severe. Parents were instructed in the use of a digital thermometer to record temperature, were asked to note use of analgesics, and were asked to respond to questions on incidence of general reactions. The diaries were collected at subsequent study visits.

A $5 \mathrm{ml}$ venous blood sample was obtained 28-42 days (standard within immunogenicity trials) after administration of the third vaccine dose, during the plateau antibody phase that follows peak antibody response at 10-14 days. ${ }^{12}{ }^{13}$ A local anaesthetic cream (Ametop; Smith and Nephew Healthcare, Hull) was applied at the site of venepuncture to minimise discomfort.

During double data entry operators were blind to the needle size allocations. We included participants in reactogenicity analyses only if the diaries were complete and they had complied with the protocol.

\section{Serological testing}

We assigned serological analyses in the order H influenzae type b, tetanus, meningococcal $\mathrm{C}$, and diphtheria antigens; fewer samples were analysed for diphtheria owing to smaller amounts of serum obtained. We used enzyme linked immunosorbent assays to measure specific serum immunoglobulin $\mathrm{G}$ against $H$ influenzae type $\mathrm{b}$ and tetanus, calibrated, respectively, against the Food and Drug Administration 1983 international standard reference serum and WHO international standard antitetanus immunoglobulin.
We carried out serogroup $\mathrm{C}$ meningococcal serum bactericidal assays against strain C11 (phenotype C:16:P1.7-1,1) using baby rabbit serum (Pel-Freeze, Rodgerson, AZ) as an exogenous complement source. We expressed serum bactericidal titres as the reciprocal of the final serum dilution giving $50 \%$ killing at 60 minutes.

A seroneutralisation assay was used to measure diphtheria antitoxin antibody levels using a micrometabolic inhibition test with reference standards (WHO international standard for diphtheria antitoxin, first international standard preparation). Laboratory staff were unaware from which group the samples came.

\section{Outcome measures}

Primary end points were measurement of the geometric mean titres of serogroup $\mathrm{C}$ meningococcal glycoconjugate antibodies and geometric mean concentrations of diphtheria, tetanus, and $H$ influenzae type $\mathrm{b}$ antibodies 28-42 days after the third vaccine dose. Secondary end points were rates of any local reaction (swelling, redness, hardness, or tenderness) at both injection sites on the evening after vaccination (six hour time point) and subsequent three evenings (days 1, 2, and 3) after each immunisation, as assessed by parents. Incidence of general reactions was also recorded. We express comparisons between needle groups as relative geometric mean titres or geometric mean concentrations for immunogenicity outcomes and as risk ratios for reactogenicity outcomes.

\section{Statistical analysis}

The trial was designed to test the hypothesis that immunogenicity after use of the wide, long needle was no lower than that after use of the narrow, short needle. We prestated an equivalence bound of a relative reduction in geometric mean titres or geometric mean concentrations of $10 \%$, above which differences would be interpreted as showing non-inferiority. We assessed statistical significance using one tailed $t$ tests on log transformed data, computing the area in the left tail beyond the $10 \%$ equivalence bound. Significance was defined at the $2.5 \%$ level. We used confidence intervals calculated from the $t$ distribution to compute the probability that an immune response was not inferior. $^{14}$

To distinguish whether difference in length or gauge affected local reaction rates, we made two pairwise comparisons of local reactogenicity rates between wide, long and narrow, short needles and wide, long and narrow, long needles. To test the significance of differences in reaction rates we used two tailed $\chi^{2}$ tests. Significance was defined at the $5 \%$ level. Differences in systemic reaction rates between the three groups were tested using a $\chi^{2}$ test with two degrees of freedom (or Fisher's exact test if event rates were low).

Presuming that immunogenicity would be $20 \%$ higher with a wide, long needle than with a narrow, short needle (using results from a study of adolescents that found a $20 \%$ difference in diphtheria and tetanus antibody levels when an intramuscular injection was compared with a subcutaneous injection ${ }^{15}$ ) we determined that 201 infants would be required per group to have $80 \%$ power of showing non-inferiority using a $10 \%$ equivalence range at a one sided $2.5 \%$ significance level, assuming that the standard deviation of the log of the serological titres was 1.0. A sample size of 201 per group would enable reductions in overall local reaction rates from $80 \%$ to $65 \%$ to be detected with $90 \%$ power.

Initially we allowed for 5\% drop out or failure to obtain a blood sample rate and aimed to recruit 633 participants. We monitored the dropout rate during the study and found it to be 
closer to $12 \%$. We therefore increased recruitment to 696 infants to maintain a target of 201 evaluable participants per group.

Statistical analyses were undertaken using SPSS version 11.5 and Stata version 9.0.

\section{Results}

Of the 696 infants enrolled, 240 were randomised to the wide, long needle, 230 to the narrow, short needle, and 226 to the narrow, long needle (fig 1). No substantial differences were found between the groups at baseline for sex, birth weight, age, or weight at each vaccination (table 1). Overall, 84 infants (12\%) were withdrawn from the study: 14 (2\%) violated the protocol, 14 (2\%) had serious local or general reactions, 5 (1\%) discontinued participation during the vaccination period, $13(2 \%)$ were lost to follow-up, and $38(5 \%)$ had inadequate blood samples or the parents refused venepuncture. Numbers were similar across groups except for serious local reactions.

\section{Wide, long needle versus narrow, short needle}

Immunogenicity-The average immune response was higher with the wide, long needle compared with the narrow, short needle, although none of the differences were statistically significant (table 2). The largest increase was observed for the meningococcal $\mathrm{C}$ vaccine where the geometric mean titre increased by $30 \%$ (95\% confidence interval $-1 \%$ to $69 \% ; \mathrm{P}=0.06)$. Significant non-inferiority of response was shown for meningococcal $\mathrm{C}$ and diphtheria, the lower limits of the $95 \%$ confidence interval being above the $10 \%$ equivalence bound (fig 2). Although H influenzae type $\mathrm{b}$ and tetanus did not show any evidence of immune response being compromised by use of the wide, long needle,
Table 1 Characteristics of 696 infants randomised to receive immunisations through one of three needle sizes

\begin{tabular}{lccc} 
Characteristic & $\begin{array}{c}\text { Wide, long } \\
\text { needle }(\mathbf{n}=\mathbf{2 4 0})\end{array}$ & $\begin{array}{c}\text { Narrow, short } \\
\text { needle }(\mathbf{n}=\mathbf{2 3 0})\end{array}$ & $\begin{array}{c}\text { Narrow, long } \\
\text { needle }(\mathbf{n}=\mathbf{2 2 6})\end{array}$ \\
\hline No (\%) boys & $107(45)$ & $138(60)$ & $115(51)$ \\
\hline Mean (SD) birth weight (g) & $3500(500)$ & $3500(400)$ & $3600(500)$ \\
\hline First vaccination: & & & \\
\hline Mean (range) age (days) & $62(56-77)$ & $62(56-76)$ & $61(55-76)$ \\
\hline Mean (SD) weight (kg) & $5.2(0.7)$ & $5.3(0.7)$ & $5.3(0.7)$ \\
\hline Second vaccination: & $\mathrm{n}=232$ & $\mathrm{n}=218$ & $\mathrm{n}=223$ \\
\hline Mean (range) age (days) & $92(82-112)$ & $91(84-113)$ & $91(83-113)$ \\
\hline Mean (SD) weight (kg) & $6.0(0.8)$ & $6.0(0.7)$ & $6.1(0.8)$ \\
\hline Third vaccination: & $\mathrm{n}=227$ & $\mathrm{n}=214$ & $\mathrm{n}=220$ \\
\hline Mean (range) age (days) & $122(111-148)$ & $121(112-143)$ & $121(111-147)$ \\
\hline Mean (SD) weight (kg) & $6.6(0.8)$ & $6.8(0.8)$ & $6.8(0.8)$ \\
\hline
\end{tabular}

Denominators differ from baseline owing to withdrawals.

this was not conclusively excluded as a possibility. The probability that immune response using the wide, long needle was not inferior was estimated to be $84 \%$ for H influenzae type b and $90 \%$ for tetanus.

Local reactogenicity to combined vaccine-On average 61\% (388) of the infants experienced local reactions to each dose of the combined vaccine. Redness occurred in $44 \%$ (279), tenderness in $27 \%$ (173), hardness in 35\% (221), and swelling in 18\% (113). Fifty four per cent (341) showed a reaction six hours after vaccination, 46\% (288) one day later, 28\% (180) two days later, and $14 \%$ (87) three days later. Infants vaccinated with the wide, long rather than the narrow, short needle experienced significantly fewer local reactions for all three doses at days 1, 2, and 3 (fig 3), with relative reductions of between $22 \%$ (6\% to $36 \%$ ) and $54 \%$

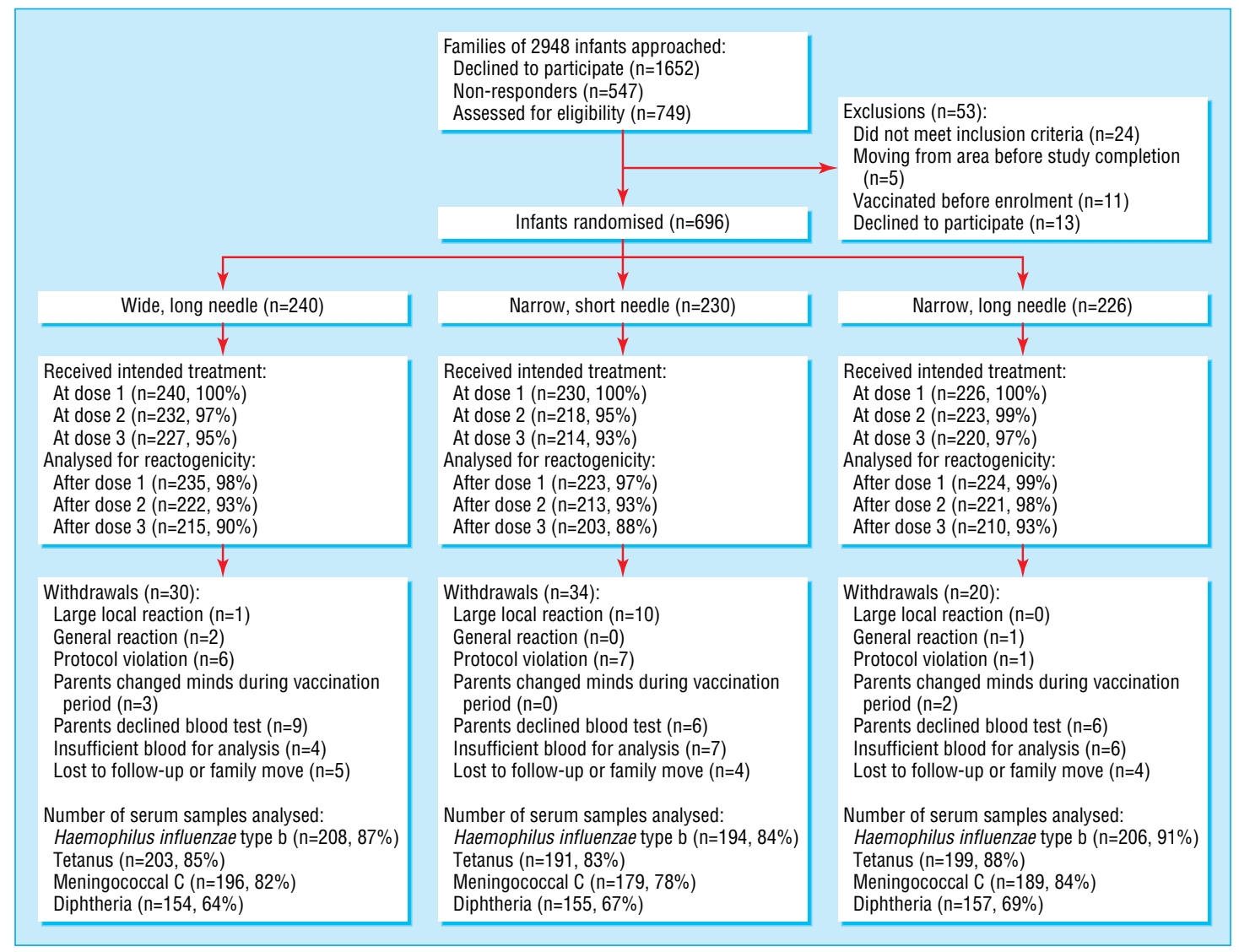

Fig 1 Trial profile 
Table 2 Vaccine immunogenicity in infants randomised to receive immunisations through one of three needle sizes

\begin{tabular}{|c|c|c|c|c|c|}
\hline \multirow{2}{*}{ Variable } & \multicolumn{3}{|c|}{ Geometric mean concentration or geometric mean titre by needle size $(95 \% \mathrm{CI})$} & \multicolumn{2}{|c|}{$\begin{array}{l}\text { Ratio of geometric mean concentration or geometric mean titre } \\
\qquad(95 \% \mathrm{Cl})^{*}\end{array}$} \\
\hline & Wide, long needle & Narrow, short needle & Narrow, long needle & $\begin{array}{l}\text { Wide, long } v \text { narrow, short } \\
\text { needle }\end{array}$ & $\begin{array}{l}\text { Narrow, long } v \text { wide, long } \\
\text { needle }\end{array}$ \\
\hline & $\mathrm{n}=196$ & $\mathrm{n}=179$ & $\mathrm{n}=189$ & - & - \\
\hline $\begin{array}{l}\text { Meningococcal C } \\
\text { vaccine† }\end{array}$ & $1262(1052$ to 1514$)$ & 973 (799 to 1184) & 1169 (972 to 1405) & $1.30(0.99$ to 1.69$)$ & $0.93(0.72$ to 1.20$)$ \\
\hline \multirow[t]{2}{*}{ SD (log) } & 1.29 & 1.28 & 1.33 & - & - \\
\hline & $\mathrm{n}=208$ & $\mathrm{n}=194$ & $\mathrm{n}=206$ & - & - \\
\hline $\begin{array}{l}\text { Haemophilus influenzae } \\
\text { type b }\end{array}$ & 3.47 (2.82 to 4.28$)$ & 3.30 (2.65 to 4.12$)$ & 4.47 (3.76 to 5.32$)$ & $1.05(0.78$ to 1.42$)$ & 1.29 (0.98 to 1.69$)$ \\
\hline \multirow[t]{2}{*}{ SD (log) } & 1.52 & 1.26 & 1.56 & - & - \\
\hline & $n=203$ & $n=191$ & $n=199$ & - & - \\
\hline Tetanus & 1.71 (1.51 to 1.93 ) & 1.68 (1.47 to 1.93$)$ & 1.63 (1.44 to 1.84$)$ & 1.01 (0.84 to 1.22$)$ & 0.96 (0.80 to 1.14$)$ \\
\hline \multirow[t]{2}{*}{ SD $(\log )$} & 0.90 & 0.88 & 0.96 & - & - \\
\hline & $\mathrm{n}=154$ & $\mathrm{n}=155$ & $\mathrm{n}=157$ & - & - \\
\hline Diphtheria & 0.24 (0.21 to 0.28$)$ & $0.21 \quad(0.18$ to 0.25$)$ & $0.22(0.20$ to 0.26$)$ & 1.13 (0.91 to 1.39$)$ & 0.93 (0.76 to 1.14$)$ \\
\hline SD (log) & 0.95 & 0.85 & 0.93 & - & - \\
\hline
\end{tabular}

SD $(\log )=$ standard deviation of measurements on natural log scale.

* Statistically significant non-inferiority corresponds to lower limit of $95 \%$ confidence interval exceeding 0.90 .

tSerogroup C meningococcal glycoconjugate vaccine.

(20\% to $73 \%)$. At the six hour assessment relative reductions were smaller $-5 \%(-12 \%$ to $20 \%)$ to $11 \%(-1 \%$ to $21 \%)$, and not statistically significant. Overall reaction rates were dominated by the high reaction rate at six hours and showed a statistically significant reduction for the first dose only (see bmj.com). Statistically significant reductions were, however, found with the wide, long needle in reactions that were evident the second day or later with relative reductions of $31 \%$ (16\% to $44 \%), 24 \%$ ( $8 \%$ to $38 \%$ ), and $24 \%$ ( $8 \%$ to $37 \%$ ) for the first, second, and third doses, respectively. The number of infants needed to vaccinate with a wide, long rather than a narrow, short needle to prevent such a reaction are 6,8 , and 8 for the three doses.

The wide, long needle also reduced the severity of local reactions. Of 11 infants withdrawn from the study due to redness and swelling covering more than two thirds of the anterolateral thigh (a contraindication to receipt of further whole cell pertussis vaccine $\left.^{1}\right) 10$ were vaccinated using the narrow, short needle and only one using the wide, long needle (Fisher's exact test, $\mathrm{P}=0.005$ )

Local reactogenicity to meningococcal $C$ vaccine-Reaction rates to the meningococcal $\mathrm{C}$ vaccine were lower than to the combined vaccine. On average 42\% (262) of participants experienced local reactions to each dose of the vaccine. Redness occurred in 27\% (171), tenderness in 18\% (114), hardness in 14\% (91), and swelling in 8\% (51). In total, 38\% (240) showed a reaction six hours after vaccination, 23\% (157) one day later, 11\% (67) two days later, and 5\% (32) three days later. No significant differences were noted between groups in overall reaction rates or in reactions evident in the second day or later (see bmj.com).
Systemic reactogenicity-No significant differences were found between the groups of infants with fever (axillary temperature $\geq 38^{\circ} \mathrm{C}$ ) after any vaccine dose. Overall analgesic use and incidence of systemic reactions did not differ significantly between groups (table 3 ).

\section{Narrow, long needle versus wide, long needle}

No significant differences were found in immunogenicity between the two longer needles (table 2). Local reaction rates seemed slightly lower with the narrow needle, although differences were small and only reached statistical significance for the overall rates of reaction for the third dose of the combined vaccine (see bmj.com) and rates on day 2 for the third dose (fig 3). No difference was found in systemic reactogenicity (table 3).

\section{Discussion}

The incidence of any local reaction after each immunisation dose in the UK schedule for infants aged 2, 3, and 4 months is significantly reduced when a wide, long $(25 \mathrm{~mm})$ needle rather than a narrow, short (16 mm) needle is used. Although only two of the four vaccine components formally showed non-inferiority, the weight of evidence clearly favoured the wide, long needle, achieving comparable, if not superior, immunogenicity to that of the narrow, short needle. If reductions in immune response up to $25 \%$ are considered to provide clinically equivalent protection, then non-inferiority would be proved for all four components.

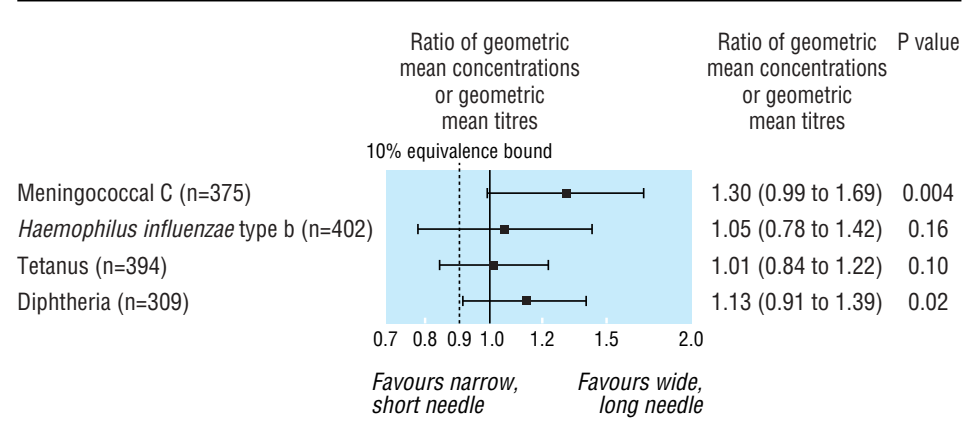

Fig 2 Ratio of antibody levels in infants immunised using different sized needles 

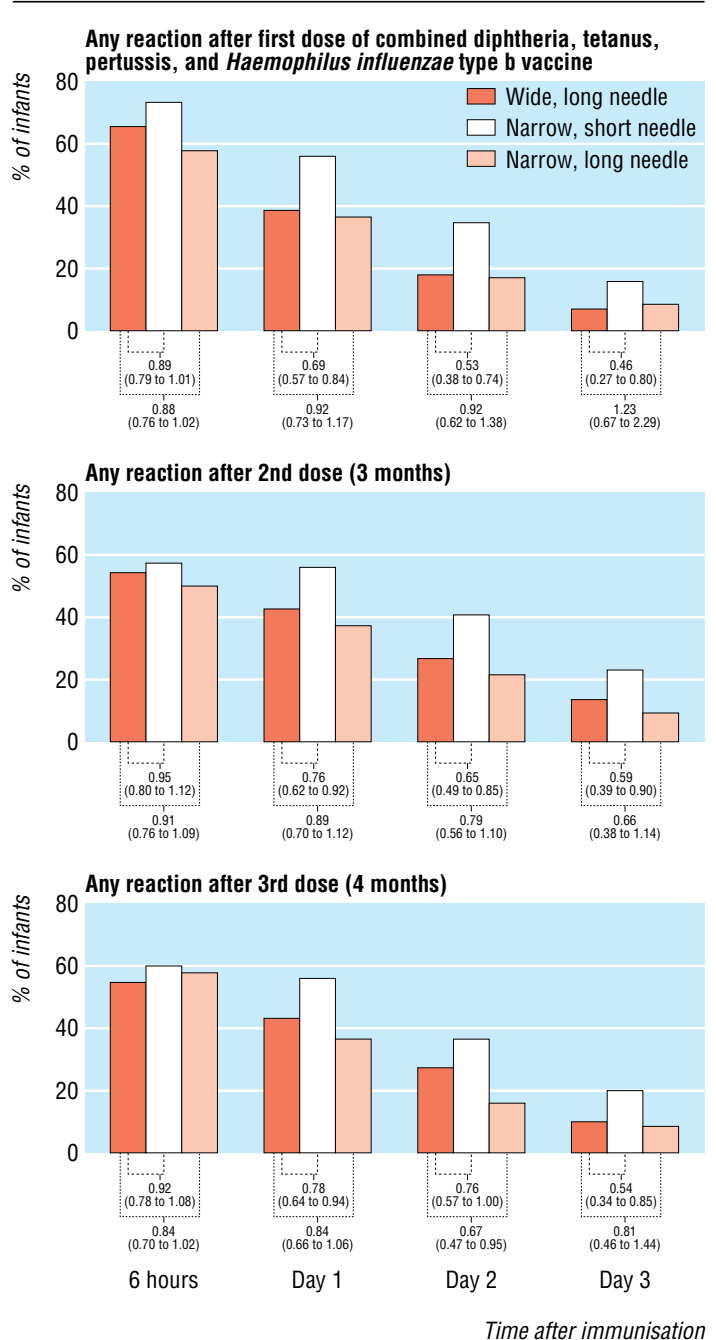

Fig 3 Rates of local reactions using three sizes of needles for immunisations in infants. Values are relative risks ( $95 \%$ confidence intervals)

This study also provides evidence that needle length, rather than gauge, is associated with reduced reactogenicity. In our previous study needle gauge had been a confounding factor. ${ }^{10}$ Some authors have suggested that the narrower 25 gauge needle might produce an injection jet under greater pressure and that this causes increased trauma and local reaction rates. ${ }^{76}$ The present study, however, provided no evidence to support this claim, with little difference being observed between needle gauges. In a systematic review recommending changing to a $25 \mathrm{~mm}$ needle, the results were limited by the inclusion of two small studies and could not separate the effect of gauge from that of length. ${ }^{17}$ These latest results would add weight to any subsequent meta-analysis.

We suggest, as stated in our previous work, that the longer 25 $\mathrm{mm}$ needle ensures delivery into an infant's thigh muscle. ${ }^{10} \mathrm{Sev}-$ eral clinical trials of vaccines in adults or adolescents have shown that intramuscular delivery minimises adverse reactions, ${ }^{15}{ }^{18}$ and a clear physiological rationale justifies its importance; poorer drainage channels in subcutaneous tissue and the propensity to retain injected material for longer seems to leave subcutaneous fat more susceptible to the adverse effects of vaccines. ${ }^{19}$ For vaccines containing aluminium adjuvant, intramuscular delivery is particularly recommended, as inadvertent subcutaneous admin-
Table 3 Systemic reactions and use of analgesics in infants after immunisations using different sized needles. Values are numbers (percentages) with reactions unless stated otherwise

\begin{tabular}{|c|c|c|c|}
\hline Variable & $\begin{array}{l}\text { Wide, long } \\
\text { needle }\end{array}$ & $\begin{array}{l}\text { Narrow, short } \\
\text { needle }\end{array}$ & $\begin{array}{l}\text { Narrow, long } \\
\text { needle }\end{array}$ \\
\hline & & & \\
\hline
\end{tabular}

Combined diphtheria, tetanus, pertussis, and Haemophilus influenzae type $\mathrm{b}$ with meningococcal C vaccine given concomitantly Any pyrexia after each

vaccination:

\begin{tabular}{lcccc}
\hline First dose & $31(13)$ & $27(12)$ & $35(16)$ & 0.51 \\
\hline Second dose & $13(6)$ & $8(4)$ & $19(9)$ & 0.11 \\
\hline Third dose & $10(5)$ & $14(7)$ & $22(10)$ & 0.07 \\
\hline Any dose & $48(20)$ & $40(18)$ & $58(26)$ & 0.10 \\
\hline $\begin{array}{l}\text { Any paracetamol after each } \\
\text { vaccination: }\end{array}$ & $147(62)$ & $138(61)$ & $131(58)$ & 0.70 \\
\hline First dose & $131(59)$ & $121(57)$ & $116(52)$ & 0.40 \\
\hline Second dose & $128(59)$ & $107(51)$ & $107(51)$ & 0.14 \\
\hline Third dose & $190(81)$ & $176(78)$ & $173(77)$ & 0.60 \\
\hline Any dose & & & & \\
\hline
\end{tabular}

Any vaccine

Systemic reactions:

\begin{tabular}{lcccc}
\hline Sleepier than usual & $195(83)$ & $187(83)$ & $181(80)$ & 0.66 \\
\hline $\begin{array}{l}\text { Vomited }>3 \text { times in } 24 \\
\text { hrs }\end{array}$ & $47(20)$ & $42(19)$ & $39(17)$ & 0.76 \\
\hline Eating less than usual & $152(65)$ & $131(58)$ & $131(58)$ & 0.27 \\
\hline $\begin{array}{l}\text { Persistent inconsolable } \\
\text { crying ( } \geq 4 \text { hours) }\end{array}$ & $4(2)$ & $2(1)$ & $5(2)$ & $0.56^{*}$
\end{tabular}
crying ( $\geq 4$ hours)

${ }^{*} \mathrm{P}$ value computed from Fisher's exact test, otherwise from $\chi^{2}$ test.

istration of this adjuvant may increase irritation or lead to lumps and nodules at the injection site. ${ }^{20}$ As injection with a $16 \mathrm{~mm}$ needle inserted at $90^{\circ}$ has been shown not to reach muscle in a significant number of infants aged 4 months, ${ }^{21}$ and as the combined diphtheria, pertussis, tetanus, and Haemophilus influenzae type $\mathrm{b}$ vaccine contains aluminium adjuvant, this may explain the differences in reactogenicity seen between needle lengths in our study.

Although few studies have compared infants' immune responses to vaccines using different needle lengths, the mechanism by which intramuscular delivery of vaccines contributes towards improved immunogenicity has been described. ${ }^{22}$ Compared with subcutaneous tissue with its poor vascularity, slower mobilisation or processing of antigens, and lack of phagocytic or antigen presenting cells, muscle has an abundant blood supply. ${ }^{22}$ In comparison in studies of adults, intramuscular delivery provided significantly improved seroconversion. ${ }^{22}{ }^{23}$ Our study provided some evidence that for meningococcal $\mathrm{C}$ vaccine the longer needle provided a better immunological response. Our results suggest that consideration of needle size is advisable when planning clinical trials of vaccines and we repeat calls for this variable to be reported to facilitate comparisons between trials. $^{24}$

After completion of this study, vaccines recommended for use in the UK immunisation schedule were changed in view of the high rates of systemic reactions attributed to vaccines containing whole cell pertussis. The combined diphtheria, pertussis, tetanus, and $H$ influenzae type b vaccine was replaced by a less reactogenic vaccine consisting of diphtheria, tetanus acellular pertussis, $H$ influenzae type $\mathrm{b}$, and inactivated polio (Pediacel; Sanofi Pasteur MSD). ${ }^{6}$ Our data cannot be directly extrapolated to this combination which is now used in the United Kingdom; however, our study implies that local reactogenicity is reduced by intramuscular delivery and there is no evidence to indicate that this would be different for other vac- 


\section{Research}

cines. Our data support use of a longer needle for improved immunogenicity of meningococcal $\mathrm{C}$ vaccine, and since this vaccine is still used in the United Kingdom, the observations remain relevant.

In conclusion, data from this study are likely to be highly relevant to settings where children receive reactogenic vaccines containing diphtheria, tetanus, and whole cell pertussis. As our data are from a population of well nourished infants, our findings may not be directly transferable to populations where most diphtheria, tetanus, and whole cell pertussis vaccines are now administered-that is, in resource poor countries with less well nourished children. Nevertheless, these data support the delivery of infant immunisations into muscle rather than subcutaneous tissue, and the longer needle size increases the likelihood that this will be achieved in all populations, irrespective of variations in injection technique. With increasing parental attention focused on the safety rather than the efficacy of vaccines, simple interventions that reduce local reactions, such as longer needles, should be welcomed. Vaccine manufacturers and national policymakers should consider needle size in recommendations for infant immunisation.

We thank the participants; research nurses Karen Stone, Helen Layton, and Tessa Waterhouse for carrying out the study visits, administering immunisations, and collecting venepuncture samples; Shamim Shah-Gallardo for distraction of infants during venepuncture; general practices of Vale of Aylesbury and North East Oxfordshire primary care trusts who participated and welcomed us into their surgeries: Vale of Aylesbury and Oxfordshire child health computer departments for mailing of invitation letters to parents; Ray Borrow and team (Health Protection Agency Meningococcal Reference Unit, Manchester Royal Infirmary) for meningococcal C serum analyses; Armelle Marais (Sanofi Pasteur MSD) for organising shipment of samples, and William Bartlett and the Global Clinical Immunology Platform team, Sanofi Pasteur US for serum analyses of diphtheria; Carly Banner (Immunology Research Laboratory, Churchill Hospital, Oxford) for serum analyses of Haemophilus influenzae type b and tetanus; David Foxcroft, Lindsey Coombes, and Jane V Appleton (Oxford Brookes University) for $\mathrm{PhD}$ research supervision; Ly-Mee Yu (Centre for Statistics in Medicine) for running the programmes for verification of double data entry and help with presentation of results; Shirley Ashmore for administrative support; and students Sarah Hall and Paul Diggle for data entry.

Contributors: LD conceived and planned the study, recruited and trained study nurses, managed data collection, undertook initial analyses, wrote the first draft of the paper, and is guarantor for the study.JJD advised on design, produced the randomisation scheme, verified initial analyses, and undertook further analyses. AJP advised on serum analyses and supervised clinical decisions relevant to the infants. LD and JJD had full access to all of the data in the study and take responsibility for the integrity of the data and the accuracy of the data analysis. All authors contributed to the manuscript.

\section{What is already known on this topic}

At age 4 months a wide, long rather than narrow, short needle for immunisation reduces local reaction

Whether this was due to needle length or gauge or could be achieved at younger immunisation ages was unknown

Effect on immunogenicity was unknown

What this study adds

Long $(25 \mathrm{~mm})$ needles provide the optimum immunisation at 2, 3 , and 4 months of age

\section{Needle length, not gauge, affects reactogenicity}

Immunogenicity is comparable between long and short (16 $\mathrm{mm}$ ) needles
Funding: This study was funded through the NHS Executive South East research and development project grant scheme. Additional support was provided by Becton Dickinson. JDD is supported by a Department of Health National Coordinating Centre for Research Capacity Development senior research fellowship in evidence synthesis.

Competing interests: $\mathrm{LD}$ has received assistance from Wyeth Vaccines and Sanofi Pasteur MSD to attend scientific meetings. AJP acts as chief investigator for clinical trials conducted on behalf of Oxford University, sponsored by vaccine manufacturers (Sanofi-Pasteur MSD, Novartis Vaccines, GlaxoSmithKline, Sanofi-Pasteur, and Wyeth Vaccines), and has received assistance from GSK vaccines, Sanofi Pasteur MSD, Novartis Vaccines, and Wyeth Vaccines to attend scientific meetings. AJP is an inventor on a patent application in the area of meningococcal B vaccines. Industry sourced consultancies or honorariums are paid directly to an independent charity or an educational fund held by the Department of Paediatrics, University of Oxford.

Ethical approval: This study was approved by the Mid and South Buckinghamshire and Oxfordshire local research ethics committees.

1 Department of Health. Immunisation against infectious diseases. London: HMSO, 1996. 2 Government Statistical Service NHS immunisation statistics 2003-04. Stat Bull 2004;16:1.

3 Office for National Statistics. Births 2004 (provisional) selected background data, England Wales. www.statistics.gov.uk/statbase/Product.asp?vlnk = 14010 (accessed 14 Apr 2006) 4 General Register Office for Scotland. Estimated population, births, stillbirths, deaths $\mathcal{E}$ marriages, number and rates by administrative area, Scotland 2004. www.groscotland.gov.uk/files/04p-p2.pdf (accessed 14 Apr 2006).

5 Northern Ireland Statistics and Research Agency. Births and deaths in Northern Ireland in 2004. www.nisra.gov.uk/uploads/publications/births_and_deaths_2004.pdf (accessed 14 Apr 2006).

6 Chief Medical Officer, Chief Nursing Officer, Chief Pharmaceutical Officer. Important changes to the childhood immunisation programme. London: Department of Health, $12 \mathrm{Jul}$ changes

7 Mayon-White RT, Moreton J. Immunizing children-a practical guide. Oxford: Radcliffe Medical, 1997

8 Grosswasser J, Kahn A, Bouche B, Hanquinet S, Perlmuter N, Hessel L. Needle length and injection technique for efficient intramuscular vaccine delivery in infants and children evaluated through an ultrasonographic determination of subcutaneous and muscle layer thickness. Pediatrics 1997;100:400-3.

9 World Health Organization. Immunization in practice: module 6 holding an immunization session. WHO/IVB/04.06. www.who.int/vaccines-diseases/epitraining SiteNew/iip/PDF/Module6.pdf (accessed 20 Jul 2006).

10 Diggle L, Deeks J. Effect of needle length on incidence of local reactions to routine immunisation in infants aged 4 months: randomised controlled trial. BMJ 2000:321:931-3.

11 Ipp MM, Gold R, Greenberg S, Goldbach M, Kupfert BB, Lloyd DD, et al. Acetaminophen prophylaxis of adverse reactions following vaccination of infants with diphtheria-pertussis-tetanus toxoids-polio vaccine. Pediatr Infect Dis J 1987;6:721-5.

12 Weir DM, Stewart J. Immunology. 8th ed. Edinburgh: Churchill Livingstone, 1997.

13 Madore DV, Johnson Kraines CL, Rothstein EP, Smith DH. Kinetics of antibody response to Haemophilus influenzae type b vaccines. Pennridge Pediatric Associates. Curr Med Res Opin 1999;15:105-12.

14 Shakespeare TP, Gebski VJ, Veness MJ, Simes J. Improving interpretation of clinical studies by use of confidence levels, clinical significance curves and risk-benefit contours. Lancet 2001:357:1349-53.

15 Mark A, Carlsson RM, Granstrom M. Subcutaneous versus intramuscular injection for booster DT vaccination of adolescents. Vaccine 1999;17:2067-72

16 Watson M. Needle length and incidence of local reactions to immunization. Needle gauge is more important than needle length. BMJ 2001;322:492.

17 Davenport JM. A systematic review to ascertain whether the standard needle is more effective than a longer or wider needle in reducing the incidence of local reaction in children receiving primary immunization. J Adv Nurs 2003;46:66-77.

18 Ruben FL, Froeschle JE, Meschievitz C, Chen K, George J, Reeves-Hoche MK, et al. Choosing a route of administration for quadrivalent meningococcal polysaccharide vaccine: intramuscular versus subcutaneous. Clin Infect Dis 2001;32:170-2.

19 Zuckerman JN. The importance of injecting vaccines into muscle. Different patients need different needle sizes. BMJ 2000;321:1237-8.

20 Bernier RH, Frank JA Jr, Nolan TF Jr. Abscesses complicating DTP vaccination. Am J Dis Child 1981;135:826-8.

21 Hick JF, Charboneau JW, Brakke DM, Goergen B. Optimum needle length for diphtheria-tetanus-pertussis inoculation of infants. Pediatrics 1989;84:136-7.

22 Shaw FE Jr, Guess HA, Roets JM, Mohr FE, Coleman PJ, Mandel EJ, et al. Effect of anatomic injection site, age and smoking on the immune response to hepatitis B vaccination. Vaccine 1989;7:425-30.

23 Reid Sanden FL, Fishbein DB, Stevens CA, Briggs DJ. Administration of rabies vaccine in the gluteal area: a continuing problem. Arch Intern Med 1991;151:821.

24 Poirier MK, Poland GA, Jacobsen RM. Parameters potentially affecting interpretation of immunogenicity and efficacy data in vaccine trials: are they adequately reported? Vaccine 1996;14:25-7.

(Accepted 29 June 2006)

doi 10.1136/bmj.38906.704549.7C

Oxford Vaccine Group, Centre for Clinical Vaccinology and Tropical Medicine, Department of Paediatrics, University of Oxford, Churchill Hospital, Oxford OX3 7LJ

Linda Diggle principal research nurse 
Research

Andrew J Pollard university lecturer in paediatric infectious diseases

Department of Public Health and Epidemiology, University of Birmingham, Birmingham
Jonathan J Deeks professor of health statistics

Correspondence to: L Diggle linda.diggle@paediatrics.ox.ac.uk $\stackrel{\text { W }}{\longrightarrow}$ 